\title{
EFFECT OF FABA BEAN - CEREALS INTERCROPPING AND NITROGEN FERTILIZATION ON THE INFESTATION BY THE LEAFMINER Liriomyza trifolii (BURGESS) ON FABA BEAN PLANTS.
}

\author{
Dalia M. B. Shawer*1 ${ }^{*}$ E.A. Taha ${ }^{1}$ and A.A. Emeran ${ }^{2}$ \\ ${ }^{1}$ Department of Economic Entomology,Faculty of Agriculture, Kafrelsheikh \\ University,33516 Kafrelsheikh, Egypt. \\ ${ }^{2}$ Department of Agricultural Botany, Faculty of Agriculture, Kafrelsheikh University, \\ 33516 Kafrelsheikh, Egypt. \\ *Corresponding Author: dalia_shamel@yahoo.com
}

\begin{abstract}
Field experiments were conducted at Kafr El-Sheikh, Egypt over two winter seasons, 2006/07 and 2007/08, to evaluate the effect of faba bean-cereals intercropping and nitrogen fertilization on leafminer, Liriomyza trifolii (Burgess) infestation on two cultivars of faba bean Vicia faba. Level of leafminer infestation increased gradually from mid-December reaching maximum levels by the end of March. Larvae population increased gradually reaching a maximum level by mid-February, and then gradually decreased and completely disappeared by the end of March. Triticale intercropping with Faba bean, cv. Giza Blancka, and nitrogen fertilization (50 kg /Feddan) resulted in the lowest susceptibility to leafminer infestation during the first and second seasons. Wheat intercropping with faba bean, cv. Giza Blancka was the best treatment to reduce the population of leafminer larvae, while barley intercropping with faba bean, cv. Yossif El-Sediek resulted in the highest leafminer population.
\end{abstract}

Keywords: Faba bean; intercropping; leafminer

\section{INTRODUCTION}

Faba bean (Vicia faba L.) is an important legume crop, used as a human food and animal feed (Rubiales, 2011). Unfortunately, faba bean plants are susceptible to the attack of numerous insect species. Leafminer (Liriomyza trifolii Burgess) is considered as a serious insect that causes considerable loss to the quantity and the quality of faba bean (Abate and Ampofo, 1996; Jung, 2014). Infestation by Liriomyza spp. can cause both direct and indirect damage, such as; disease transmission, seedling death, crop reduction, fruit damage, and reduction of the aesthetic value of ornamental plants, in addition to plant quarantine problems (Abdul Rassoul and Al-Saffar, 2013). Difference in plant susceptibility and plant resistance to the insect attack has been reported for most faba bean cultivars (Hannou and Hegazi, 1996; Abdallah et al. 1996, 2000; El-Khouly et al., 1997; Mohamed and Salman, 2001; Abdel-Samad and Ahmed, 2007 and Awadalla et al., 2013 and 2014) . As leafminer will continue to be important pest of high value crops, insecticides will continue to be an important component for leafminer management, therefore it is imperative to continue to refine the use of insecticides that target leafminer. Improving application, timing and methods will help to conserve insecticides susceptibility and maintain efficacy by mitigating the evolution of resistance (Reitz et al., 2013).

Generally, most of the control approaches are partially effective and sometimes inconsistent and affected by the environmental conditions. Intercropping is the simultaneous cultivation of more than one crop species in close association to take the advantage of biodiversity, competition and complement between the two crops (Boudreau, 1993). The simultaneous cultivation of legumes and cereals increased the protein content of the legume crop, and the forage nutritional value of the cereal crop (Strydhorst et al., 2008). The beneficial effect of intercropping and nitrogen fertilization for disease control has often been acknowledged (Finckh et al., 2000; Mundt, 2002).

This research is focusing on using some integrated pest managements methods to control leafminer on faba bean, in a way to reduce or cease insecticides use that target leafminer. The objective of this study is to test various combined treatments of cultivars, fertilization, and intercropping on the leafminer L. trifolii.

\section{MATERIALS AND METHODS}

Field experiments were carried out at Amya region, Kafr El-Sheikh, Egypt during two successive winter seasons of 2006/07 and 2007/08. Two faba bean cultivars; Giza Blancka (Large seeds) and Yossif ElSediek (small seeds) were cultivated in ten treatments each. In each season, an area of about $400 \mathrm{~m}^{2}$ was prepared and divided into 80 plots of about $4 \mathrm{~m}^{2}$ each. Every plot was separated from other plots with two uncultivated rows (about $55 \mathrm{~cm}$ both). Treatments were evaluated in 2 cultivars (Giza Blancka and Yossif ElSediek) $\times 5$ cropping systems (faba bean alone, and intercropped with oat Avena sativa L., cv. Aspen, barley Hordeum vulgare L., cv. Cory, wheat Triticum durum Desf., cv. Meridiano, or triticale Triticosecale wittm, cv. Peñarroya $) \times 2$ fertilization levels $(0$ and $50 \mathrm{~kg}$ N/Feddan) completely randomized block design with 4 replicates. All faba bean seeds were sown on October $15^{\text {th }}$ of both seasons. Seeds were planted ( 2 seeds/hill) at $20 \mathrm{~cm}$ apart between hills.

Leafminers Liriomyza trifolii (Burgess) assessment was started one month after sowing (November $15^{\text {th }}$ ), and was performed at 15-day intervals until faba bean plants reached the maturity stage by March $31^{\text {st }}$. Ten plants per plot were randomly chosen to 
count the total number of plant leaflets, and number of infested leaflets. The percentage of infestation were calculated according to the following equations;

\section{$\frac{\text { Number of infested leaflets } \times 100}{\text { Total number of leaflets }}$}

To determine the population densities of $L$. trifolii larvae, a sample of 25 leaflets per replicate was randomly chosen form upper, middle; and lower parts of faba bean plants. The chosen leaflets were placed in polyethylene bags and transferred to the lab for inspection.

Data were analyzed using the general linear model procedure of SAS 9.2 (SAS, Inc., 2002), and means separation was performed using Duncan multiple range test (DMRT) (Duncan, 1955).

\section{RESULTS AND DISCUSSION}

\section{Infestation percentage}

Results in Tables 1 and 2 showed that the infestation percentage increased gradually in all treatments towards the end of both seasons. Most data showed that Yousef El-Seddiek cultivar is more susceptible for leafminers infestation than Giza Blancka cultivar. Oat intercropping with Yousef El-eddiek cultivar at both nitrogen levels ( 0 and $50 \mathrm{Kg} \mathrm{N} /$ Feddan) increased infestation percentage significantly in comparison with Giza Blancka cultivar during both seasons. A significant difference was also noticed between both cultivars when intercropping with Triticale at $50 \mathrm{Kg} \mathrm{N} /$ Feddan in both seasons. Intercropping triticale with Giza Blancka cultivar at 50 $\mathrm{Kg} \mathrm{N} /$ Feddan showed the highest and significant infestation percentage in comparison with all other treatments, except with the treatment of oat intercropping with Giza Blancka cultivar at $50 \mathrm{Kg}$ $\mathrm{N} /$ Feddan, in both seasons.

The analysis of variance of these data generally showed a significant effect of both the cultivar and the intercropping, as well as the combination effect of cultivar, fertilization, and intercropping in both seasons (Table 5).

The variation among faba bean cultivars in susceptibility to leafminer under the Egyptian environmental conditions may be related to the morphological and the anatomical characteristics, such as leaf shape and color, solidness of the leaflets, and cell wall thickness, as well as the physiological characteristics, such as repellent and attractants of the plants, and some other factors, such as the environmental conditions, location, and planting date (Hassanein, 1989; Abdallah et al., 1996; Hannou and Hegazi,1996; El-Khouly et al., 1997; Abdallah et al., 2000; Mohamed and Salman, 2001; Abdel-Samad and Ahmed, 2007; El-Samahy, 2008; Salman et al., 2015).

The difference in leafminer infestation percentage due to intercropping may be due to the physiological characters of the intercropped plants, such as repellent and/or attractants. In this regard, Liebman and Dyck (1993) reported that intercropping can be an effective ecological method to control pests, diseases and weeds via natural competition that allows for more efficient resources utilization. Many African farmers traditionally intercrop maize or sorghum with legumes to increase crop production, achieving better returns on fertilizers, pesticides, energy and manpower resources (Fernandez-Aparicio et al., 2008). Many authors applied intercropping on legumes to reduce either infestation with pests or infection with diseases. Intercropping faba bean with lupine, fenugreek, or Egyptian clover markedly reduced its infestation with root parasite Orobanche crenata Forsk (Bakheit et al., 2002) or the obligate parasite Orobanche foetida (Kharrat and Halila, 2005). Intercropping of maize or sorghum with legumes was used to reduce infection by witchweed Striga hermonthica (Oswald et al., 2002). Also, intercropping of legumes with oat (Fernandez-Aparicio et al., 2007) or with fenugreek (Fernandez-Aparicio et al., 2008) reduced the infestation of legumes by root parasite Orobanche crenata Forsk. Intercropping of bean with maize significantly reduced the incidence and the severity levels of bean common bacterial blight and rust, compared with sole cropping (Fininsa, 1996).

\section{Population fluctuation of leafminer}

Results in Tables 3 and 4 showed that the larvae of leafminer were found on all treatments during the same time of the growing season on December 15 of both seasons. The population of larvae gradually increased with the progress of the season, and reached the maximum level on February 15, and then decreased gradually until disappeared by the end of March in both seasons. The number of larvae per 25 leaflets counted on Yousef El-Sediek cultivar was generally higher than that on Giza Blancka cultivar, and a significant difference between both cultivars was noticed at the control treatment, intercropping with triticale at $0 \mathrm{Kg}$ $\mathrm{N} /$ Feddan, and intercropping with barley, wheat, or triticales at $50 \mathrm{Kg} \mathrm{N} /$ Feddan in both seasons.

Like the infestation percentage, the analysis of variance of results of both seasons showed a significant effect of the cultivar, and the interaction of cultivar, fertilization and intercropping on the number of larvae per 25 leaflets, but no significant effect was noticed in regards to the intercropping (Table 5).

These results are in agreements with the findings of Hannou and Hegazi (1996), El-Khouly et al. (1997), Abdallah et al. (2000), Mohamed and Salman (2001), Ebadah et al. (2006), Abdel-Samad and Ahmed (2007) and El-Samahy (2008) who concluded that the preference of leafminer to a cultivar than another may be related to cell wall thickness and solidness of the leaflets, as well as the repellent effect or the attraction of the plant to the insect. 
J. Plant Prot. and Path., Mansoura Univ., Vol.7(1), January, 2016

Table (1):Effect of intercropping and nitrogen fertilization on the percentage of leafminer $L$. trifolii infestation on Faba bean plants during the first season 2006/07.

\begin{tabular}{|c|c|c|c|c|c|c|c|c|c|c|c|}
\hline \multicolumn{3}{|c|}{ Treatments } & \multicolumn{9}{|c|}{ Infestation (\%) } \\
\hline \multirow[b]{2}{*}{ Variety } & \multirow{2}{*}{$\begin{array}{c}\text { Nitrogen } \\
\text { fertilization }\end{array}$} & \multirow[b]{2}{*}{ Intercropping } & \multicolumn{2}{|c|}{ December 2006} & \multicolumn{2}{|c|}{ January 2007} & \multicolumn{2}{|c|}{ February 2007} & \multicolumn{2}{|c|}{ March 2007} & \multirow[b]{2}{*}{ Mean } \\
\hline & & & 15 & 31 & 15 & 31 & 15 & 28 & 15 & 31 & \\
\hline \multirow{10}{*}{$\begin{array}{l}\text { Yousef } \\
\text { El-Sediek }\end{array}$} & \multirow{5}{*}{$0 \mathrm{Kg} /$ Feddan } & None & $23.40^{\mathrm{abc}}$ & $38.31^{\mathrm{abc}}$ & $41.20^{\text {ghi }}$ & $56.19^{\mathrm{ab}}$ & $69.19^{\mathrm{ab}}$ & $89.79^{\mathrm{a}}$ & $92.31^{\text {abcd }}$ & $100.00^{\mathrm{a}}$ & $63.80^{\mathrm{ab}}$ \\
\hline & & Oat & $22.11^{\text {cdef }}$ & $43.34^{\mathrm{ab}}$ & 45.65 abcde & $51.44^{\text {abcd }}$ & $69.98^{\mathrm{ab}}$ & $88.68^{\mathrm{ab}}$ & $90.17^{\text {bce }}$ & $99.33^{\mathrm{ab}}$ & $63.84^{\mathrm{ab}}$ \\
\hline & & Barley & $22.35^{\text {cde }}$ & $42.23^{\mathrm{ab}}$ & $43.07^{\text {cdefghi }}$ & $56.63^{\mathrm{ab}}$ & $69.19^{\mathrm{ab}}$ & $89.79^{\mathrm{a}}$ & $89.64^{\text {cde }}$ & $99.08^{\mathrm{ab}}$ & $64.00^{\mathrm{abc}}$ \\
\hline & & Wheat & $22.66^{\text {bcd }}$ & $42.27^{\mathrm{ab}}$ & $45.14^{\text {abcdef }}$ & $57.23^{\mathrm{a}}$ & 65.28 abcde & $88.58^{\mathrm{ab}}$ & 91.37 abcde & $100.00^{\mathrm{a}}$ & $64.07^{\mathrm{a}}$ \\
\hline & & Triticale & $22.75^{\text {abcd }}$ & $42.48^{\mathrm{ab}}$ & $44.31^{\text {bcdefg }}$ & $55.49^{\mathrm{abc}}$ & $68.88^{\mathrm{abc}}$ & $89.23^{\mathrm{a}}$ & $90.76^{\text {bcde }}$ & $100.00^{\mathrm{a}}$ & $64.24^{\mathrm{a}}$ \\
\hline & \multirow{5}{*}{$50 \mathrm{Kg} /$ Feddan } & None & $21.17^{\text {defg }}$ & $43.79^{\mathrm{a}}$ & $46.38^{\text {abcd }}$ & $47.86^{\mathrm{de}}$ & $69.83^{\mathrm{ab}}$ & $87.96^{\mathrm{abc}}$ & $89.64^{\text {cde }}$ & $99.25^{\mathrm{ab}}$ & $63.24^{\mathrm{abc}}$ \\
\hline & & Oat & $19.12^{\mathrm{h}}$ & $35.04^{\mathrm{c}}$ & $42.22^{\text {defghi }}$ & $57.49^{\mathrm{a}}$ & $69.19^{\mathrm{ab}}$ & $89.79^{\text {a }}$ & 91.74 abcde & $100.00^{\mathrm{a}}$ & $63.07^{\mathrm{abc}}$ \\
\hline & & Barley & $21.90^{\text {cdef }}$ & $39.23^{\mathrm{abc}}$ & $42.05^{\text {efghi }}$ & $57.74^{\mathrm{a}}$ & $62.26^{\text {def }}$ & $88.90^{\mathrm{ab}}$ & $90.90^{\text {bcde }}$ & $99.18^{\mathrm{ab}}$ & $62.77^{\text {abcd }}$ \\
\hline & & Wheat & $20.65^{\text {efgh }}$ & $43.79^{\mathrm{a}}$ & $47.84^{\mathrm{a}}$ & $49.23^{\text {cde }}$ & $68.37^{\mathrm{abc}}$ & $84.21^{\mathrm{c}}$ & $89.71^{\text {cde }}$ & $98.70^{\mathrm{b}}$ & $62.81^{\text {abcd }}$ \\
\hline & & Triticale & $23.15^{\text {abc }}$ & $43.66^{\mathrm{a}}$ & $42.59^{\text {defghi }}$ & $49.56^{\text {cd }}$ & $68.83^{\text {abc }}$ & $88.41^{\mathrm{ab}}$ & $95.00^{\mathrm{a}}$ & $100.00^{\mathrm{a}}$ & $63.90^{\mathrm{ab}}$ \\
\hline \multirow{10}{*}{$\begin{array}{l}\text { Giza } \\
\text { Blancka }\end{array}$} & \multirow{5}{*}{$0 \mathrm{Kg} /$ Feddan } & None & $24.59^{\mathrm{ab}}$ & $43.16^{\mathrm{ab}}$ & $46.38^{\text {abcd }}$ & $50.37^{\text {bcd }}$ & $65.37^{\text {abcde }}$ & $87.81^{\mathrm{abc}}$ & 91.04 abcde & $100.00^{\mathrm{a}}$ & $63.59^{\mathrm{abc}}$ \\
\hline & & Oat & $19.86^{\mathrm{gh}}$ & $37.24^{\text {bc }}$ & $43.79^{\text {bcdefgh }}$ & $50.23^{\text {bcd }}$ & $61.99^{\text {def }}$ & $89.14^{\mathrm{a}}$ & 91.37 abcde & $100.00^{\mathrm{a}}$ & $61.70^{\text {cd }}$ \\
\hline & & Barley & $24.67^{\mathrm{a}}$ & $40.34^{\mathrm{abc}}$ & $44.21^{\text {bcdefg }}$ & $46.44^{\mathrm{def}}$ & $59.35^{\mathrm{f}}$ & $89.88^{\mathrm{a}}$ & $94.24^{\mathrm{ab}}$ & $100.00^{\mathrm{a}}$ & $62.39^{\text {abcd }}$ \\
\hline & & Wheat & $23.08^{\mathrm{abc} d}$ & $41.82^{\mathrm{ab}}$ & 45.41 abcde & $50.41^{\text {bcd }}$ & $63.00^{\text {cdef }}$ & $89.17^{\text {a }}$ & $95.00^{\mathrm{a}}$ & $100.00^{\mathrm{a}}$ & $63.49^{\mathrm{abc}}$ \\
\hline & & Triticale & $23.53^{\mathrm{abc}}$ & $44.28^{\mathrm{a}}$ & $40.07^{\mathrm{i}}$ & $51.68^{\text {abcd }}$ & $66.46^{\text {abcd }}$ & $86.87^{\mathrm{abc}}$ & $95.00^{\mathrm{a}}$ & $100.00^{\mathrm{a}}$ & $63.49^{\mathrm{abc}}$ \\
\hline & \multirow{5}{*}{$50 \mathrm{Kg} /$ Feddan } & None & $23.60^{\mathrm{abc}}$ & $39.96^{\mathrm{abc}}$ & $43.13^{\text {cdefghi }}$ & $41.25^{\mathrm{fg}}$ & $64.14^{\text {bcdef }}$ & 88.18 abc & $92.96^{\mathrm{abc}}$ & $100.00^{\mathrm{a}}$ & $61.65^{\text {cd }}$ \\
\hline & & Oat & $20.41^{\mathrm{fgh}}$ & $40.31^{\mathrm{abc}}$ & $41.84^{\text {fghi }}$ & $47.38^{\mathrm{de}}$ & $66.08^{\text {abcde }}$ & $84.75^{\mathrm{bc}}$ & $88.65^{\mathrm{de}}$ & $98.65^{\mathrm{b}}$ & $61.01^{\mathrm{de}}$ \\
\hline & & Barley & $22.73^{\text {bcd }}$ & $41.576^{\mathrm{ab}}$ & $43.98^{\text {bcdefgh }}$ & $56.54^{\mathrm{ab}}$ & $64.80^{\text {abcdef }}$ & $87.82^{\mathrm{abc}}$ & $88.20^{\mathrm{e}}$ & $100.00^{\mathrm{a}}$ & $63.21^{\mathrm{abc}}$ \\
\hline & & Wheat & $21.76^{\text {cdef }}$ & $42.23^{\mathrm{ab}}$ & 42.91 defghi & $43.02^{\text {efg }}$ & $64.19^{\text {bcdef }}$ & $89.51^{\mathrm{a}}$ & $92.69^{\text {abcd }}$ & $100.00^{\mathrm{a}}$ & $62.04^{\text {bcd }}$ \\
\hline & & Triticale & $28.00^{\mathrm{h}}$ & $38.93^{\mathrm{abc}}$ & $40.61^{\mathrm{hi}}$ & $39.51^{\mathrm{h}}$ & $60.22^{\text {ef }}$ & $87.95^{\mathrm{abc}}$ & $90 / 29^{\text {bcde }}$ & $100.00^{\mathrm{a}}$ & $60.69^{\mathrm{e}}$ \\
\hline \multicolumn{3}{|c|}{ Significance } & $* *$ & $*$ & $* *$ & $* *$ & $* *$ & $*$ & $* *$ & $*$ & $* *$ \\
\hline
\end{tabular}

Means of each column followed by the same letter are not significantly different at the $5 \%$ level according to Duncan 's Multiple Range Test.

- * and ** indicate $\mathbf{P}<0.05$ and $\mathbf{P}<0.01$, respectively.

Table(2):Effect of intercropping and nitrogen fertilization on the percentage of leafminer $L$. trifolii infestation on Faba bean plants during the second season 2007/08.

\begin{tabular}{|c|c|c|c|c|c|c|c|c|c|c|c|}
\hline \multicolumn{3}{|c|}{ Treatments } & \multicolumn{9}{|c|}{ Infestation (\%) } \\
\hline \multirow[b]{2}{*}{ Variety } & \multirow{2}{*}{$\begin{array}{c}\text { Nitrogen } \\
\text { fertilization }\end{array}$} & \multirow[b]{2}{*}{ Intercropping } & \multicolumn{2}{|c|}{ December 2007} & \multicolumn{2}{|c|}{ January 2008} & \multicolumn{2}{|c|}{ February 2008} & \multicolumn{2}{|c|}{ March 2008} & \multirow[t]{2}{*}{ Mean } \\
\hline & & & 15 & 31 & 15 & 31 & 15 & 29 & 15 & 31 & \\
\hline \multirow{10}{*}{$\begin{array}{l}\text { Yousef } \\
\text { El-Sediek }\end{array}$} & \multirow{5}{*}{$0 \mathrm{Kg} /$ Feddan } & None & $24.63^{\mathrm{ab}}$ & $40.33^{\mathrm{abc}}$ & $43.37^{\mathrm{efg}}$ & $59.15^{\mathrm{a}}$ & $74.50^{\mathrm{a}}$ & $92.64^{\mathrm{abc}}$ & $97.17^{\text {abcd }}$ & $100.00^{\mathrm{a}}$ & $66.47^{\mathrm{ab}}$ \\
\hline & & Oat & $23.27 \mathrm{cdef}$ & $45.63^{\mathrm{ab}}$ & $48.58^{\mathrm{ab}}$ & $54.14^{\mathrm{bc}}$ & $73.66^{\mathrm{ab}}$ & $93.35^{\mathrm{ab}}$ & $94.91^{\text {cde }}$ & $99.40^{\mathrm{ab}}$ & $66.62^{\mathrm{a}}$ \\
\hline & & Barley & $23.53^{\text {cde }}$ & $44.45^{\mathrm{ab}}$ & $45.34^{\text {bcdefg }}$ & $59.61^{\mathrm{a}}$ & $70.63^{\text {abcd }}$ & $92.26^{\mathrm{abc}}$ & $94.36^{\text {cde }}$ & $99.13^{\mathrm{ab}}$ & $66.16^{\mathrm{abc}}$ \\
\hline & & Wheat & $23.85^{\text {bcd }}$ & $44.49^{\mathrm{ab}}$ & $47.51^{\text {abcd }}$ & $61.03^{\mathrm{a}}$ & $68.72^{\text {abcde }}$ & $93.24^{\mathrm{ab}}$ & 96.18 abcde & $100.00^{\mathrm{a}}$ & $66.88^{\mathrm{a}}$ \\
\hline & & Triticale & $23.95^{\mathrm{abc}}$ & $44.72^{\mathrm{ab}}$ & $46.64^{\text {abcde }}$ & $58.41^{\mathrm{ab}}$ & $72.51^{\mathrm{abc}}$ & $93.93^{\mathrm{a}}$ & $95.54^{\text {bcde }}$ & $100.00^{\mathrm{a}}$ & $66.96^{\mathrm{a}}$ \\
\hline & \multirow{5}{*}{$50 \mathrm{Kg} /$ Feddan } & None & $22.28^{\text {defg }}$ & $46.09^{\mathrm{a}}$ & $49.12^{\mathrm{a}}$ & $49.59^{\mathrm{cd}}$ & $73.51^{\mathrm{ab}}$ & $92.59^{\mathrm{abc}}$ & $94.36^{\text {cde }}$ & $99.40^{\mathrm{ab}}$ & $65.87^{\text {abc }}$ \\
\hline & & Oat & $20.13^{\mathrm{h}}$ & $36.89^{\mathrm{c}}$ & $44.44^{\text {cdefg }}$ & $60.52^{a}$ & $72.83^{\mathrm{ab}}$ & $94.52^{\mathrm{a}}$ & 96.57 abcde & $100.00^{\mathrm{a}}$ & $65.74^{\mathrm{abc}}$ \\
\hline & & Barley & $23.05^{\text {cdef }}$ & $42.29^{\mathrm{abc}}$ & $44.27^{\text {cdefg }}$ & $60.78^{a}$ & $65.54^{\text {def }}$ & $93.58^{\mathrm{ab}}$ & $95.69^{\text {bcde }}$ & $99.40^{\mathrm{ab}}$ & $65.58^{\text {abcd }}$ \\
\hline & & Wheat & $21.74^{\text {efgh }}$ & $46.09^{\mathrm{a}}$ & $48.87^{\mathrm{ab}}$ & $52.32^{\mathrm{c}}$ & $71.97^{\mathrm{abc}}$ & $88.64^{\mathrm{c}}$ & $94.43^{\text {cde }}$ & $98.77^{\mathrm{b}}$ & $65.35^{\text {abcd }}$ \\
\hline & & Triticale & $24.37^{\mathrm{ab}}$ & $45.96^{\mathrm{a}}$ & $44.84^{\text {cdefg }}$ & $52.17^{\mathrm{c}}$ & $72.46^{\mathrm{abc}}$ & $93.07^{\mathrm{ab}}$ & $100.00^{\mathrm{a}}$ & $100.00^{\mathrm{a}}$ & $66.61^{\mathrm{a}}$ \\
\hline \multirow{10}{*}{$\begin{array}{l}\text { Giza } \\
\text { Blancka }\end{array}$} & \multirow{5}{*}{$0 \mathrm{Kg} /$ Feddan } & None & $25.88^{\mathrm{ab}}$ & $45.43^{\mathrm{ab}}$ & $48.82^{\mathrm{ab}}$ & $53.02^{\mathrm{c}}$ & $68.81^{\text {abcde }}$ & $92.43^{\mathrm{abc}}$ & $95.84^{\text {abcde }}$ & $100.00^{\mathrm{a}}$ & $66.28^{\mathrm{ab}}$ \\
\hline & & Oat & $20.90^{\text {gh }}$ & $39.20^{\mathrm{bc}}$ & $46.10^{\text {abcdef }}$ & $52.87^{\mathrm{c}}$ & $65.25^{\text {def }}$ & $93.83^{\mathrm{a}}$ & $96.18^{\text {abcde }}$ & $100.00^{\mathrm{a}}$ & $64.29^{\mathrm{cd}}$ \\
\hline & & Barley & $25.97^{\mathrm{a}}$ & $42.47^{\mathrm{abc}}$ & $46.54^{\text {abcde }}$ & $48.93^{\mathrm{cd}}$ & $62.47^{\mathrm{f}}$ & $94.61^{\mathrm{a}}$ & $99.20^{\mathrm{ab}}$ & $100.00^{\mathrm{a}}$ & $65.02^{\text {abcd }}$ \\
\hline & & Wheat & $24.29^{\mathrm{abc}}$ & $44.02^{\mathrm{ab}}$ & $47.80^{\mathrm{abc}}$ & $53.06^{\mathrm{c}}$ & $66.32^{\text {cdef }}$ & $93.86^{\mathrm{a}}$ & $100.00^{\mathrm{a}}$ & $100.00^{\mathrm{a}}$ & $66.17^{\text {abc }}$ \\
\hline & & Triticale & $24.77^{\mathrm{ab}}$ & $46.61^{\mathrm{a}}$ & $42.18^{\mathrm{g}}$ & $53.65^{\mathrm{bc}}$ & $69.96^{\text {abcd }}$ & $91.44^{\mathrm{abc}}$ & $100.00^{\mathrm{a}}$ & $100.00^{\mathrm{a}}$ & $66.08^{\text {abc }}$ \\
\hline & \multirow{5}{*}{$50 \mathrm{Kg} /$ Feddan } & None & $24.84^{\mathrm{ab}}$ & $42.06^{\mathrm{abc}}$ & $45.40^{\text {bcdefg }}$ & $43.16^{\mathrm{e}}$ & $67.51^{\text {bcdef }}$ & $92.83^{\mathrm{abc}}$ & $97.86^{\mathrm{abc}}$ & $100.00^{\mathrm{a}}$ & $64.21^{\mathrm{cd}}$ \\
\hline & & Oat & $21.48^{\mathrm{fgh}}$ & $42.43^{\mathrm{abc}}$ & $44.04^{\mathrm{defg}}$ & $50.63^{c}$ & $69.55^{\text {abcde }}$ & $89.21^{\mathrm{bc}}$ & $93.32^{\mathrm{de}}$ & $98.70^{\mathrm{b}}$ & $63.67^{\mathrm{de}}$ \\
\hline & & Barley & $23.93^{\text {bcd }}$ & $43.76^{\mathrm{ab}}$ & $46.29^{\text {abcde }}$ & $59.52^{\mathrm{a}}$ & $68.21^{\text {abcdef }}$ & $92.44^{\mathrm{abc}}$ & $92.84^{\mathrm{e}}$ & $100.00^{\mathrm{a}}$ & $65.87^{\text {abc }}$ \\
\hline & & Wheat & $22.90^{\text {cde }_{f}}$ & $44.45^{\mathrm{ab}}$ & $44.93^{\text {cdefg }}$ & $45.29^{\mathrm{de}}$ & $67.57^{\text {abcdef }}$ & $94.22^{\mathrm{a}}$ & $97.57^{\text {abcd }}$ & $100.00^{\mathrm{a}}$ & $64.62^{\text {bcd }}$ \\
\hline & & Triticale & $20.29^{\mathrm{h}}$ & $40.98^{\mathrm{abc}}$ & $42.67^{\mathrm{fg}}$ & $41.59^{\mathrm{e}}$ & $63.39^{\text {ef }}$ & $92.58^{\mathrm{abc}}$ & $95.04^{\text {bcde }}$ & $100.00^{\mathrm{a}}$ & $62.07^{\mathrm{e}}$ \\
\hline \multicolumn{3}{|c|}{ Significance } & $* *$ & $*$ & $* *$ & $* *$ & $* *$ & $*$ & $* *$ & * & $* *$ \\
\hline
\end{tabular}

Means of each column followed by the same letter are not significantly different at the 5\% level according to Duncan 's Multiple Range Test.

- * and ** indicate $\mathbf{P}<0.05$ and $\mathbf{P}<0.01$, respectively. 
Dalia M. B. Shawer et al.

Table (3): Effect of intercropping and nitrogen fertilization on the number of $L$. trifolii larvae/25 leaflets of Faba bean plants during the first season 2006/07.

\begin{tabular}{|c|c|c|c|c|c|c|c|c|c|c|c|}
\hline \multicolumn{3}{|c|}{ Treatments } & \multicolumn{9}{|c|}{ Number of larvae / 25 leaflets } \\
\hline \multirow{2}{*}{ Variety } & \multirow{2}{*}{$\begin{array}{c}\text { Nitrogen } \\
\text { fertilization }\end{array}$} & \multirow{2}{*}{ Intercropping } & \multicolumn{2}{|c|}{ December 2006} & \multicolumn{2}{|c|}{ January 2007} & \multicolumn{2}{|c|}{ February 2007} & \multicolumn{2}{|c|}{ March 2007} & \multirow[t]{2}{*}{ Mean } \\
\hline & & & 15 & 31 & 15 & 31 & 15 & 28 & 15 & 31 & \\
\hline \multirow{10}{*}{$\begin{array}{l}\text { Yousef El- } \\
\text { Sediek }\end{array}$} & \multirow{5}{*}{$0 \mathrm{Kg} /$ Feddan } & None & $8.81^{\mathrm{ab}}$ & $23.02^{\mathrm{a} \text { bc }}$ & $23.27^{\mathrm{abc}}$ & $35.94^{\text {abcdefg }}$ & $78.00^{\text {abcd }}$ & $39.67^{\mathrm{bcd}}$ & $29.79^{\mathrm{a}}$ & $5.42^{\mathrm{a}}$ & $30.49^{\mathrm{b}}$ \\
\hline & & Oat & $9.08^{\mathrm{a}}$ & $21.26^{\text {bcde }}$ & $25.19^{\mathrm{a}}$ & $31.59^{\mathrm{fgh}}$ & $86.13^{\mathrm{a}}$ & $35.75^{\text {cdefg }}$ & $18.20^{\mathrm{b}}$ & $2.71^{\mathrm{ab}}$ & $28.74^{\mathrm{bc}}$ \\
\hline & & Barley & $8.56^{\mathrm{abc}}$ & $22.35^{\text {abcd }}$ & $23.02^{\mathrm{abc}}$ & $37.78^{\text {abcde }}$ & $64.46^{\text {cde }}$ & $32.50^{\text {defg }}$ & $10.83^{\text {bcd }}$ & $0.00^{\mathrm{b}}$ & $24.94^{\text {defg }}$ \\
\hline & & Wheat & $7.78^{\text {bcd }}$ & $20.31^{\text {cde }}$ & $22.21^{\mathrm{abc}}$ & $33.54^{\text {cdefgh }}$ & $83.55^{\mathrm{ab}}$ & $29.79^{\mathrm{fg}}$ & $15.84^{\mathrm{bc}}$ & $0.00^{\mathrm{b}}$ & $26.63^{\text {cde }}$ \\
\hline & & Triticale & $8.56^{\mathrm{abc}}$ & $22.34^{\text {abcd }}$ & $22.21^{\mathrm{abc}}$ & $35.10^{\text {bcdefgh }}$ & $88.83^{a}$ & $41.17^{\mathrm{bc}}$ & $10.83^{\text {bcd }}$ & $0.00^{\mathrm{b}}$ & $28.63^{\mathrm{bc}}$ \\
\hline & \multirow{5}{*}{$50 \mathrm{Kg} /$ Feddan } & None & $8.30^{\text {abcd }}$ & $21.62^{\text {abcde }}$ & $24.92^{\mathrm{a}}$ & $40.94^{\mathrm{a}}$ & $76.92^{\text {abcd }}$ & $44.42^{\mathrm{bc}}$ & $16.25^{\mathrm{b}}$ & $0.00^{\mathrm{b}}$ & $29.17^{\mathrm{bc}}$ \\
\hline & & Oat & $8.56^{\mathrm{abc}}$ & $22.34^{\mathrm{abcd}}$ & $20.58^{\mathrm{c}}$ & $37.54^{\text {abcde }}$ & $78.44^{\text {abcd }}$ & $41.71^{\mathrm{bc}}$ & $10.91^{\text {bcd }}$ & $0.00^{\mathrm{b}}$ & $27.51^{\mathrm{cd}}$ \\
\hline & & Barley & $8.91^{\text {ab }}$ & $23.25^{\mathrm{abc}}$ & $23.83^{\mathrm{ab}}$ & $39.00^{\mathrm{abc}}$ & $87.21^{\mathrm{a}}$ & $53.63^{\mathrm{a}}$ & $32.50^{\mathrm{a}}$ & $2.71^{\mathrm{ab}}$ & $33.88^{\mathrm{a}}$ \\
\hline & & Wheat & $9.34^{\mathrm{a}}$ & $24.37^{\mathrm{a}}$ & $23.34^{\mathrm{abc}}$ & $38.61^{\text {abcd }}$ & $86.13^{\mathrm{a}}$ & $45.77^{b}$ & $13.54^{\mathrm{bc}}$ & $2.71^{\mathrm{ab}}$ & $30.48^{\mathrm{b}}$ \\
\hline & & Triticale & $9.08^{\mathrm{a}}$ & $23.70^{\mathrm{ab}}$ & $20.85^{\text {bc }}$ & $37.78^{\text {abcde }}$ & $84.50^{\mathrm{ab}}$ & $40.63^{\text {bcd }}$ & $8.13^{\text {cde }}$ & $0.00^{\mathrm{b}}$ & $28.08^{\mathrm{bc}}$ \\
\hline \multirow{10}{*}{$\begin{array}{l}\text { Giza } \\
\text { Blancka }\end{array}$} & \multirow{5}{*}{$0 \mathrm{Kg} /$ Feddan } & None & $8.30^{\text {abcd }}$ & $21.59^{\text {abcde }}$ & $22.48^{\mathrm{abc}}$ & $29.64^{\mathrm{h}}$ & $71.50^{\mathrm{a} b c d}$ & $30.88^{\text {efg }}$ & $10.83^{\text {bcd }}$ & $2.71^{\mathrm{ab}}$ & $24.74^{\text {defg }}$ \\
\hline & & Oat & $8.75^{\mathrm{ab}}$ & $22.85^{\mathrm{abc}}$ & $20.58^{c}$ & $33.64^{\text {cdefgh }}$ & $81.76^{\mathrm{abc}}$ & $37.92^{\text {bcd }}$ & $10.83^{\text {bcd }}$ & $0.00^{\mathrm{b}}$ & $27.04^{\text {cde }}$ \\
\hline & & Barley & $7.26^{\mathrm{d}}$ & $18.96^{\mathrm{e}}$ & $20.64^{\mathrm{c}}$ & $33.15^{\text {defgh }}$ & $62.81^{\mathrm{de}}$ & $36.30^{\text {cde }}$ & $10.81^{\mathrm{bcd}}$ & $0.00^{\mathrm{b}}$ & $23.74^{\mathrm{fg}}$ \\
\hline & & Wheat & $8.30^{\text {abcd }}$ & $21.67^{\text {abcde }}$ & $22.75^{\mathrm{abc}}$ & $34.71^{\text {cdefgh }}$ & $81.33^{a b c}$ & $38.46^{\text {bcde }}$ & $8.13^{\text {cde }}$ & $0.00^{\mathrm{b}}$ & $26.92^{\text {cd }}$ \\
\hline & & Triticale & $8.30^{\text {abcd }}$ & $21.67^{\text {abcde }}$ & $22.21^{\mathrm{abc}}$ & $33.15^{\text {defgh }}$ & $73.13^{\mathrm{abcd}}$ & $30.88^{\text {efg }}$ & $2.71^{\mathrm{e}}$ & $0.00^{\mathrm{b}}$ & $24.01^{\mathrm{efg}}$ \\
\hline & \multirow{5}{*}{$50 \mathrm{Kg} /$ Feddan } & None & $8.82^{\mathrm{ab}}$ & $23.02^{\mathrm{abc}}$ & $22.48^{\mathrm{abc}}$ & $40.50^{\mathrm{ab}}$ & $77.46^{\text {abcd }}$ & $27.62^{g}$ & $10.83^{\mathrm{bcd}}$ & $0.00^{\mathrm{b}}$ & $26.34^{\text {cdef }}$ \\
\hline & & Oat & $7.26^{\mathrm{d}}$ & $18.96^{\mathrm{e}}$ & $22.39^{\mathrm{ab}}$ & $29.74^{\text {gh }}$ & $74.75^{\text {abcd }}$ & $43.33^{\mathrm{bc}}$ & $16.25^{\mathrm{b}}$ & $0.00^{\mathrm{b}}$ & $26.59^{\text {cde }}$ \\
\hline & & Barley & $7.78^{\text {bcd }}$ & $20.31^{\text {cde }}$ & $23.29^{\mathrm{abc}}$ & $37.05^{\text {abcdef }}$ & $80.71^{\mathrm{abc}}$ & $39.00^{\text {bcde }}$ & $8.13^{\text {cde }}$ & $0.00^{\mathrm{b}}$ & $27.03^{\mathrm{cd}}$ \\
\hline & & Wheat & $8.56^{\mathrm{abc}}$ & $22.34^{\text {abcd }}$ & $21.40^{\mathrm{bc}}$ & $31.20^{\mathrm{gh}}$ & $53.07^{\mathrm{e}}$ & $38.46^{\text {bcde }}$ & $8.13^{\text {cde }}$ & $0.00^{\mathrm{b}}$ & $22.90^{\mathrm{g}}$ \\
\hline & & Triticale & $7.52^{\mathrm{cd}}$ & $19.75^{\mathrm{de}}$ & $22.48^{\mathrm{abc}}$ & $32.66^{\text {efgh }}$ & $67.71^{\text {bcd }}$ & $41.71^{\mathrm{bc}}$ & $5.42^{\mathrm{de}}$ & $0.00^{\mathrm{b}}$ & $24.66^{\text {defg }}$ \\
\hline \multicolumn{3}{|c|}{ Significance } & $* *$ & $* *$ & $* *$ & $* *$ & $* *$ & $* *$ & $* *$ & $* *$ & $* *$ \\
\hline
\end{tabular}

Means of each column followed by the same letter are not significantly different at the $5 \%$ level according to Duncan 's Multiple Range Test.

- ** indicate $\mathbf{P}<\mathbf{0 . 0 1}$

Table (4): Effect of intercropping and nitrogen fertilization on the number of $L$. trifolii larvae/25 leaflets of Faba bean plants during the second season 2007/08.

\begin{tabular}{|c|c|c|c|c|c|c|c|c|c|c|c|}
\hline \multicolumn{3}{|c|}{ Treatments } & \multicolumn{9}{|c|}{ Number of larvae / 25 leaflets } \\
\hline \multirow{2}{*}{ Variety } & \multirow{2}{*}{$\begin{array}{c}\text { Nitrogen } \\
\text { fertilization }\end{array}$} & \multirow{2}{*}{ Intercropping } & \multicolumn{2}{|c|}{ December 2007} & \multicolumn{2}{|c|}{ January 2008} & \multicolumn{2}{|c|}{ February 2008} & \multicolumn{2}{|c|}{ March 2008} & \multirow{2}{*}{ Mean } \\
\hline & & & 15 & 31 & 15 & 31 & 15 & 29 & 15 & 31 & \\
\hline \multirow{10}{*}{$\begin{array}{l}\text { Yousef } \\
\text { El-Sediek }\end{array}$} & \multirow{5}{*}{$0 \mathrm{Kg} /$ Feddan } & None & $9.04 \mathrm{ab}$ & $23.60 \mathrm{abc}$ & $23.56 \mathrm{abc}$ & 36.40abcdef & $80.00 \mathrm{abcd}$ & $40.69 \mathrm{bcd}$ & $30.56 \mathrm{a}$ & $5.56 \mathrm{a}$ & $31.18 \mathrm{~b}$ \\
\hline & & Oat & $9.30 \mathrm{a}$ & 21.79 bcde & $25.82 \mathrm{a}$ & 32.40 ef & $88.33 \mathrm{a}$ & $36.67 \mathrm{cdefg}$ & $18.67 \mathrm{~b}$ & $2.78 \mathrm{ab}$ & $29.47 \mathrm{bc}$ \\
\hline & & Barley & $8.78 \mathrm{abc}$ & 22.91 abcd & $23.59 \mathrm{bc}$ & 38.75 abcd & $66.11 \mathrm{cde}$ & $33.33 \mathrm{defg}$ & $11.11 \mathrm{bcd}$ & $0.00 \mathrm{~b}$ & $25.57 \mathrm{defg}$ \\
\hline & & Wheat & $7.97 \mathrm{bcd}$ & 20.82 cde & $22.77 \mathrm{abc}$ & 34.40 cdef & $85.69 \mathrm{ab}$ & $30.56 \mathrm{fg}$ & $16.24 \mathrm{ab}$ & $0.00 \mathrm{~b}$ & $27.31 \mathrm{cde}$ \\
\hline & & Triticale & $8.77 \mathrm{abc}$ & 22.90 abcd & $22.77 \mathrm{abc}$ & 36.00abcdef & $91.11 \mathrm{a}$ & $42.22 \mathrm{bc}$ & $11.11 \mathrm{bcd}$ & $0.00 \mathrm{~b}$ & $29.36 \mathrm{bc}$ \\
\hline & \multirow{5}{*}{$50 \mathrm{Kg} /$ Feddan } & None & $8.50 \mathrm{abcd}$ & 22.16abcde & $25.54 \mathrm{a}$ & $42.00 \mathrm{a}$ & 78.89abcd & $45.56 \mathrm{bc}$ & $16.67 \mathrm{~b}$ & $0.00 \mathrm{~b}$ & $29.92 b c$ \\
\hline & & Oat & $8.77 \mathrm{abc}$ & 22.90 abcd & $21.10 \mathrm{c}$ & $38.50 \mathrm{abcd}$ & $80.45 \mathrm{abcd}$ & $42.78 \mathrm{bc}$ & $11.19 \mathrm{bcd}$ & $0.00 \mathrm{~b}$ & $28.21 \mathrm{~cd}$ \\
\hline & & Barley & $9.13 \mathrm{ab}$ & $23.83 \mathrm{abc}$ & $24.43 \mathrm{ab}$ & $40.00 \mathrm{abc}$ & $89.44 \mathrm{a}$ & $55.00 \mathrm{a}$ & $33.33 \mathrm{a}$ & $2.78 \mathrm{ab}$ & $34.74 \mathrm{a}$ \\
\hline & & Wheat & $9.57 \mathrm{a}$ & $24.98 \mathrm{a}$ & $23.93 \mathrm{abc}$ & $39.60 \mathrm{abcd}$ & $88.33 \mathrm{a}$ & $46.94 \mathrm{~b}$ & $13.89 \mathrm{bc}$ & $2.78 \mathrm{ab}$ & $31.25 \mathrm{~b}$ \\
\hline & & Triticale & $9.31 \mathrm{a}$ & $24.29 \mathrm{ab}$ & $21.38 \mathrm{bc}$ & $38.75 \mathrm{abcd}$ & $86.67 \mathrm{ab}$ & $41.67 \mathrm{bcd}$ & $8.33 \mathrm{cde}$ & $0.00 \mathrm{~b}$ & $28.80 \mathrm{bc}$ \\
\hline \multirow{10}{*}{$\begin{array}{l}\text { Giza } \\
\text { Blancka }\end{array}$} & \multirow{5}{*}{$0 \mathrm{Kg} /$ Feddan } & None & 8.50abcd & 22.13abcde & $23.04 \mathrm{abc}$ & $30.40 \mathrm{f}$ & 73.33abcd & $31.67 \mathrm{efg}$ & $11.11 \mathrm{bcd}$ & $2.78 \mathrm{ab}$ & $25.37 \mathrm{defg}$ \\
\hline & & Oat & $8.97 \mathrm{ab}$ & $23.43 \mathrm{abc}$ & $21.10 \mathrm{c}$ & 34.50 cdef & $83.86 \mathrm{abc}$ & 38.89 cde & $11.11 \mathrm{bcd}$ & $0.00 \mathrm{~b}$ & $27.73 \mathrm{~cd}$ \\
\hline & & Barley & $7.44 \mathrm{~d}$ & $19.43 \mathrm{~d}$ & $21.16 \mathrm{c}$ & 34.00 cdef & $64.42 \mathrm{de}$ & 37.22 cdef & $11.08 \mathrm{bcd}$ & $0.00 \mathrm{~b}$ & $24.34 \mathrm{fg}$ \\
\hline & & Wheat & $8.51 \mathrm{abcd}$ & 22.21abcde & $23.32 \mathrm{abc}$ & 35.60 bcdef & $83.42 \mathrm{abc}$ & 39.44 cde & 8.33 cde & $0.00 \mathrm{~b}$ & $27.60 \mathrm{~cd}$ \\
\hline & & Triticale & $8.51 \mathrm{abcd}$ & 22.20abcde & $22.77 \mathrm{abc}$ & 34.00 cdef & 75.00abcd & 31.67 efg & $2.78 \mathrm{e}$ & $0.00 \mathrm{~b}$ & $24.62 \mathrm{efg}$ \\
\hline & \multirow{5}{*}{$50 \mathrm{Kg} /$ Feddan } & None & $9.04 \mathrm{ab}$ & $23.60 \mathrm{abc}$ & $23.04 \mathrm{abc}$ & $41.60 \mathrm{ab}$ & 79.44abcd & $28.33 \mathrm{~g}$ & $11.11 \mathrm{bcd}$ & $0.00 \mathrm{~b}$ & $27.02 \mathrm{cdef}$ \\
\hline & & Oat & $7.44 \mathrm{~d}$ & $19.43 \mathrm{abc}$ & $22.95 \mathrm{abc}$ & $30.50 \mathrm{f}$ & 76.67abcd & $44.44 \mathrm{bc}$ & $16.67 \mathrm{~b}$ & $0.00 \mathrm{~b}$ & $27.26 \mathrm{cde}$ \\
\hline & & Barley & $7.98 \mathrm{bcd}$ & 20.82 cde & $23.87 \mathrm{abc}$ & 38.00 abcde & $82.78 \mathrm{abc}$ & $40.00 \mathrm{cde}$ & $8.33 \mathrm{cde}$ & $0.00 \mathrm{~b}$ & $27.72 \mathrm{~cd}$ \\
\hline & & Wheat & $8.77 \mathrm{abc}$ & 22.90 abcd & $21.93 \mathrm{bc}$ & $32.00 \mathrm{ef}$ & $54.44 \mathrm{e}$ & $39.44 \mathrm{cde}$ & $8.33 \mathrm{cde}$ & $0.00 \mathrm{~b}$ & $23.48 \mathrm{~g}$ \\
\hline & & Triticale & $7.71 \mathrm{~cd}$ & $20.24 \mathrm{de}$ & $23.04 \mathrm{abc}$ & $33.50 \mathrm{def}$ & 69.44bcde & $42.78 \mathrm{bc}$ & $5.56 \mathrm{de}$ & $0.00 \mathrm{~b}$ & $25.28 \mathrm{defg}$ \\
\hline \multicolumn{3}{|c|}{ Significance } & $* *$ & $* *$ & $* *$ & $* *$ & $* *$ & $* *$ & $* *$ & $* *$ & $* *$ \\
\hline
\end{tabular}

Means of each column followed by the same letter are not significantly different at the $5 \%$ level according to Duncan 's Multiple Range Test.

** indicate $\mathbf{P}<0.01$. 
Table (5): Analysis of variance of the effect of cultivar, intercropping, and fertilization on average leafminer L. trifolii infestation percentage and larvae number / 25 leaflets during the two successive seasons 2006/07 and 2007/08.

\begin{tabular}{|l|c|c|c|c|}
\hline \multirow{2}{*}{ Source of Variance } & Infestation percentage & \multicolumn{2}{|c|}{ Larvae number / 25 leave } \\
\cline { 2 - 5 } & $\mathbf{2 0 0 6 / 0 7}$ & $\mathbf{2 0 0 7 / 0 8}$ & $\mathbf{2 0 0 6 / 0 7}$ & $\mathbf{2 0 0 7 / 0 8}$ \\
\hline Cultivar & $*$ & $*$ & $* *$ & $\mathrm{n}$ \\
\hline Intercropping & $* *$ & $* *$ & $\mathrm{~ns}$ & $\mathrm{~ns}$ \\
\hline Fertilization & $\mathrm{ns}$ & $\mathrm{ns}$ & $\mathrm{ns}$ & $\mathrm{ns}$ \\
\hline Cultivar $\times$ Intercropping & $\mathrm{ns}$ & $\mathrm{ns}$ & $\mathrm{ns}$ & $\mathrm{ns}$ \\
\hline Cultivar $\times$ Fertilization & $\mathrm{ns}$ & $\mathrm{ns}$ & $* *$ & $*$ \\
\hline Intercropping $\times$ Fertilization & $\mathrm{ns}$ & $\mathrm{ns}$ & $*$ & $*$ \\
\hline Cultivar $\times$ Intercropping $\times$ Fertilization & $* *$ & $* *$ & & \\
\hline
\end{tabular}

* Significant at $P \leq 0.05, * *$ Significant at $P \leq 0.01,{ }^{\text {ns }}$ non-significant.

\section{CONCLUSION}

This study was part of integrated pest management research project that aimed to reduce leafminer infestation, and subsequently crop quantity and quality of faba bean. Economic damages from leafminer can be mitigated if proactive management decisions were considered to reduce the likelihood of inducing severe outbreaks of leafminer. It can be concluded that Giza Blancka cultivar showed better results than Yousef El-Sediek in terms of less plant susceptibility to leafminer infestation, and the number of larvae counted on the infested plants. Intercropping with triticale with the addition of $50 \mathrm{Kg} \mathrm{N} /$ Feddan showed the best results in that regard, which might be our recommendation to reduce leafminer effects on faba bean. Leafminer management will best be accomplished through research on, and implementation of, comprehensive integrated pest management strategies.

\section{REFERENCES}

Abate, T., Ampofo, J.K., 1996. Insect pests of bean in Africa. Their ecology and management. Ann. Rev. Entomol. 41, 45-73

Abdallah, F.E., Bleih, S.B., El-Shouny, K.Y., 1996. Varietal resistance, cultural practice and chemical control against the broad bean leafminer fly, Liriomyza congesta (Beck.) infestation under natural field conditions. AlAzhar J. Agric. Res. 23, 195-215.

Abdallah, F.E., Bleih, S.B., El-Naggar, M.A., Gameih, G.N., 2000. Susceptibility of some faba bean varieties, insecticides alternative and yield loss estimation due to Liriomyza congesta (Beck.) and Aphis carccivora Koch. Menufia J. Agric. Res. 23, 195-215.

Abdel-Samad, S., Ahmed, M.A.S., 2007. Population fluctuation of Aphis craccivora and Liriomyza trifolii and their endoparasitoids on certain faba bean varieties. XVI Int. Plant Prot. Cong. $15^{\text {th }}-$ $18^{\text {th }}$ Oct.

Abdul Rassoul, M.S. and Al-Saffar, H.H. 2013. Survey of the genus Liriomza Mik. (Diptera//; Agromyzisdae) of Iraq.Advances in Bioresearch (ABR),4(3):92-94.
Awadalla, S. S. ; Abdallah, F. E. and El-Mashaly Nora R. (2013). Influence of varieties on main insect pests attacking faba bean plants. J. Plant Prot. And Path., Mansoura Univ. 4(6):581589.

Awadalla, S. S. ; Abdallah, F. E. and El-Mashaly Nora R. (2014). Population density of main insect pests attacking faba bean plants as influenced by sowing dates. Glob. J. Agric. Food Safety Sci., 1(2) :169-177.

Bakheet, B.R.; Allam, A.Y.; Gallal, A.H. 2002. Intercropping Faba bean with some legume crops to control Orobanche crenata. Acta Agronomica Hungarica. 50 (1): 1-6.

Boudreau, M.A., 1993. Effect of intercropping beans with maize on the severity of angular leaf spot of beans in Kenya. Plant Pathol. 42, 16e25.

Duncan, B.D., 1955. Multiple Range and Multiple F. Test. Biometrics 11: 1- 42.

Ebadah, I.A., Mahmoud, Y.A., Moawad, S.S., 2006. Susceptibility of some faba bean cultivars to field infestation with some insect pests. Res. J. Agric. Biol. Sci. 2 (6), 537-540.

El-Khouly, A.S., Metwally, M.M., Salem, R.M., Helal, H.M., El-Mezaien, A.B., 1997. Field studies on broad bean leafminer, Liriomyza congesta (Beck.) A. varietals resistance. B. Spatial distribution of larvae under conditions of Kafrelsheikh Governorate, Egypt. Egypt J. Agric. Res.75 (4), 961-967.

El-Samahy, M.F., 2008. Studies on faba bean aphid, Aphis craccivora and faba bean leafminer Liriomyza trifolii and their natural enemies at Kafrelsheikh Governorate. Ph.D. Thesis, Fac. Agric. Tanta Univ. 167pp.

Fernandez-Aparicio, M. Sillero, J.C.; Rubiales, D. 2007. Intercropping with cereals reduces infection by Orobouche crenata in legumes. Crop protection 26, 1166-1172.

Fernandez-Aparicio, M., Emeran, A.A. and Rubiales, D. 2008. Control of Orobanche crenata in legumes intercropped with fenugreek (Trigonella foenumgraecum). Crop Protection 27: 653-659. 
Finckh, M.R.; Gacek, E.S.; GGoyeau, H.; Lannou, C.; Merz, U.; Mundt, C.C.; Munk, L. Nadziak, J; Newton, A.C.; de Vallavielle-Pope, C.; Wolfe, M.S. 2000. Cereal variety and species mixtures in practice, with emphasis on disease resistance. Agronomie 20: 813-837.

Fininsa, C., 1996. Effect of intercropping bean with maize on bean common bacterial blight and rust diseases. Int. J. pest management 42 (1), 51-54.

Hannou, M.A., Hegazi, E.M., 1996. Effects of faba bean cultivars and potassium fertilization on population of Liriomyza spp. (Dipetra: Agromyzidae). J Agric. Sci. Alex. Univ. 21 (12), 4565-4574.

Hassanein, S.M., 1989. Population dynamics and seasonal abundance of certain leaf-pests attacking some crops at Khattara region, Sharkia Governorate, Egypt. Zagazig J. Agric. Res. 16 (1), 137-152.

Jung, C. 2014. Leafminer fly Liriomzya spp. (Diptera: Agromyzidae) and their natural enemies on potato crop in Korea. Proceedings of the Entomology 2014 conference, Entomological Society of America, November 16-19, Portland, Oregon, USA.

Kharrat, M.; Halila, M.H. 2005. Evaluation d'autres moyens de lute contre 1' Orobanche foetida poir. Sur Vicia faba L. In: Kroschel, J., Abderabihi, M., Betz, H. (Eds.), Advances in Parasitic weed control at On-farm level, Vol. II. Joint Action to control Orobanche in the WANA Region. Margraf Verlag, Weikersheim, Germany, pp. 259-264. (Abst).

Liebman, M., Dyck, E., 1993. Crop rotation and intercropping strategies for weed management. Ecol. Appl. 3 (1), 92-122.
Mohamed, A.M., Salman, F.A., 2001. Susceptibility of some broad bean varieties to natural infestation with Aphis craccivora Koch. and Liriomyza trifolii (Burgess) at upper Egypt. Assiut J. Agric. Sci., 32 (1), 167-173.

Mundt, C.C. 2002. Performance of wheat cultivars and cultivar mixtures in the presence of Cephalosporium stripe. Crop Prot. 1:93-99.

Oswald, A.; Ransom, J.K.; Kroschel, J.; Sauerborn, J. 2002. Intercropping controls Striga in maize based farming systems. Crop Protection. 21, 367374.

Reitz, S.R.; Gao, Y., Lei, Z. 2013. Insecticide use and the ecology of invasive Liriomyza leafminer management. In: Trdan, S. "Insecticides Development of safer and more effective technologies". ISBN 978-953-51-0958-7. Ebook; http://dx.doi.org/10.5772/53874

Rubiales, D. 2011. There is hope of Faba bean cultivation. Grain Legumes, No 56, April 2011, Page 4.

Salman, A.M.A.; Abd El-Rahman, A.G.; El-Maghraby, T.Q.H. 2015. Sudceptability of some Faba bean (Vicia faba L.) varaities to infestation with Cowpea Aphid, Aphis craccivora (Koch) and Liromyza trifolii (Burgess) in Toshka region, Aswan, Egypt. Middle East Journal of Agriculture Research, 4 (1): 31-36.

SAS institute, 2002. SAS/STAT Guide for Personal Computers. Version (6 Cary, NC, SAS Institute).

Strydhorst S.M., King J.R., Lopetinsky K.J., Harker K.N. (2008): Forage potential of intercropping barley with faba bean, lupin, or field pea. Agronomy Journal, 100: 182-190.

\footnotetext{
تأثير التحميل والتسـميد النيتروجيني على الإصـابة بصـانعة الأنفـق Liriomyza trifolii (Burgess) على نباتات الفول البلائي

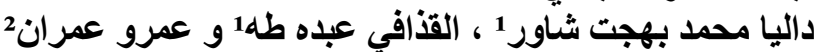

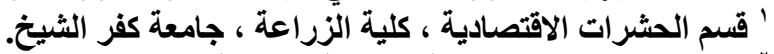

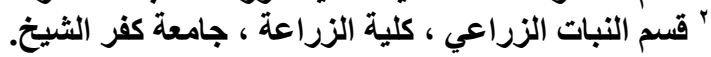

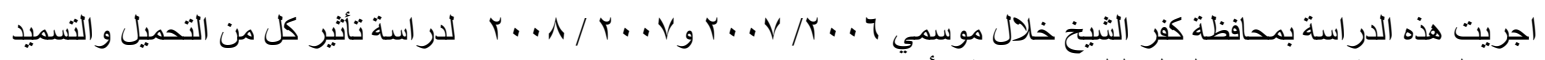

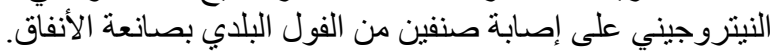

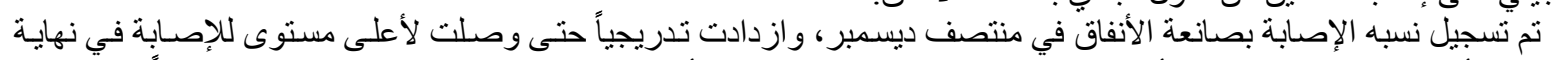

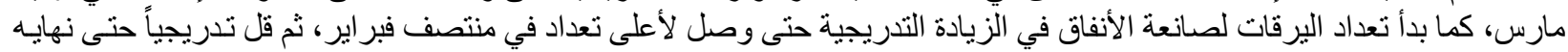

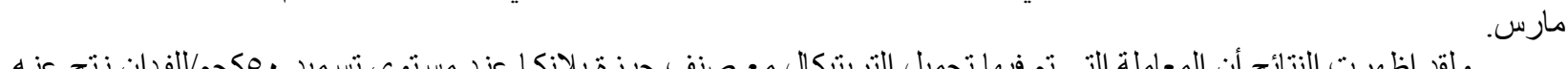
ولقد اظهرت النتائج أن المعاملة التي تم فيها تحميل التريتيكال مع صنف جيزة بلانكا عند مستوي تسميد • مكجم/للفدان نتج عنه

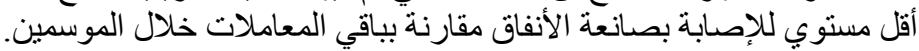

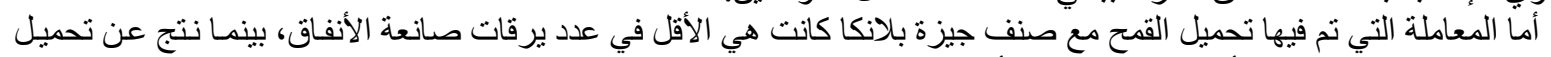

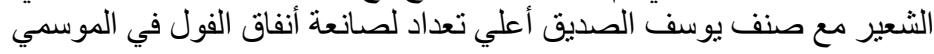

